\title{
Overall and abdominal obesity indicators had different association with central arterial stiffness and hemodynamics independent of age, sex, blood pressure, glucose, and lipids in Chinese community- dwelling adults
}

This article was published in the following Dove Press journal:

Clinical Interventions in Aging

26 November 2013

Number of times this article has been viewed

\section{Shihui Fu' \\ Leiming Luo' \\ Ping $\mathrm{Ye}^{\prime}$ \\ Yuan Liu' \\ Bing Zhu' \\ Jin Zheng' \\ Yongyi Bai' \\ Jie $\mathrm{Bai}^{2}$}

'Department of Geriatric Cardiology, ${ }^{2}$ Department of Clinical Biochemistry, Chinese People's Liberation Army General Hospital, Beijing, People's Republic of China
Correspondence: Leiming Luo Department of Geriatric Cardiology, Chinese People's Liberation Army General Hospital, Fuxing

Road 28, Haidian District, Beijing I00853, People's Republic of China

Tel +86 I0 88626362

Email Ileim@sina.com
Objective: Limited large sample studies have specially compared overall and abdominal obesity in relation to central arterial stiffness and hemodynamics in community-dwelling adults, especially in the People's Republic of China. This study aimed to compare the relationship between an overall obesity indicator (body mass index [BMI]), an abdominal obesity index (waist circumference [WC]), and central arterial stiffness and hemodynamics, independent of age, sex, blood pressure, glucose, and lipids, in Chinese community-dwelling adults.

Methods: For 2,624 adults in this study, anthropometric indices, such as BMI and WC, were measured. Central arterial stiffness was assessed by carotid-femoral pulse wave velocity (cfPWV). Central hemodynamics was represented by central pulse pressure (cPP).

Results: Both overall and abdominally obese adults were older, with significantly higher cfPWV, cPP, peripheral pulse pressure (pPP), fasting blood glucose (FBG), and low-density lipoprotein-cholesterol (LDL-C), and significantly lower high-density lipoprotein-cholesterol (HDL-C). After adjusting for age and sex, both the overall and abdominally obese individuals had independently higher pPP, FBG, and LDL-C levels, and lower HDL-C level. The overall obese individuals had independently higher cPP, but not cfPWV, after adjusting for age and sex, while the abdominally obese individuals had independently higher cfPWV, but not cPP. After adjusting for age, sex, pPP, FBG, LDL-C, and HDL-C, WC, but not BMI, was independently correlated with cfPWV, and BMI, but not WC, was independently associated with cPP. Age, sex, pPP, FBG, and HDL-C levels have independent association with cfPWV. Age, sex, pPP, but not FBG and HDL-C levels, have independent association with cPP.

Conclusion: The abdominal obesity index (WC), rather than the overall obesity indicator (BMI), was related to central arterial stiffness, independent of age, sex, blood pressure, glucose and lipids, while the overall obesity indicator (BMI), rather than the abdominal obesity indicator (WC), was independently correlated with central hemodynamics. Age, sex, and blood pressure were independently associated with central arterial stiffness and hemodynamics, but blood glucose and lipids were independently associated with central arterial stiffness, rather than hemodynamics.

Keywords: carotid-femoral pulse wave velocity, central pulse pressure, body mass index, waist circumference

\section{Introduction}

Obesity is a determinant factor in the development of hypertension, diabetes, dyslipidemia, and cardiovascular damage. ${ }^{1-4}$ Central arterial stiffness, an effective reflection 
of arterial structure, is assessed by carotid-femoral pulse wave velocity (cfPWV), and central hemodynamics is evaluated by central pulse pressure (cPP), a non-invasive representation of arterial function; both are important predictors of cardiovascular morbidity and mortality. ${ }^{5-7}$ Several studies have analyzed the relationship between obesity and central arterial stiffness and hemodynamics. ${ }^{8-11}$ For example, similar relationship between aortic stiffness and body weight have been documented in both the Cardiovascular Health Study and the Health ABC study. ${ }^{12,13}$ But, limited studies have specially compared overall and abdominal obesity in relation to central arterial stiffness and hemodynamics. A Spanish study of 305 subjects, by Recio-Rodriguez et al, has shown that waist circumference (WC) correlates better than body mass index (BMI) with arterial stiffness, measured by pulse wave velocity (PWV). ${ }^{14}$ To our knowledge, few large sample studies have elucidated whether such relationship differs in community-dwelling adults, especially in the People's Republic of China; a large sample investigation directed at Chinese community-dwelling adults is imperative. Thus, the main objective of this study was to compare possible relationship of an overall obesity indicator (BMI), and an abdominal obesity index (WC), with parameters that measure central arterial stiffness (PWV) and hemodynamics (cPP), independent of age, sex, blood pressure, glucose, and lipids, in Chinese community-dwelling adults.

\section{Subjects and methods Study population}

This is an analysis of baseline data from a large health checkup program of community-dwelling residents in Beijing, People's Republic of China. A stratified cluster sampling design was used in this survey. In the first stage of sampling, three districts (Fengtai, Shijingshan, and Daxing) were selected from 18 districts in Beijing. In the second stage of sampling, four communities were selected from these districts. In the third stage of sampling, individual participants were selected from these communities. The inclusion period started in May 2007 and ended in July 2009. A total of 2,624 subjects were included in the analysis. Each subject was a permanent resident of Han origin, more than 18 years old.

\section{Anthropometric measurements}

All anthropometrics and blood pressure were measured by physicians in the Chinese People's Liberation Army General Hospital, who had been well trained by the research team. Weight was measured on a digital scale while subjects wore light clothing with no shoes. Height was determined using a wall-mounted measuring tape, subjects standing with no shoes. BMI was calculated as weight $(\mathrm{kg})$ divided by height squared $\left(\mathrm{m}^{2}\right)$. WC was measured in standing subjects using a flexible tape, midway between the lowest rib and the iliac crest. Overall obesity was defined as BMI greater than or equal to $28 \mathrm{~kg} / \mathrm{m}^{2}$. Abdominal obesity was considered as WC greater than or equal to $85 \mathrm{~cm}$, in males, and WC greater than or equal to $80 \mathrm{~cm}$, in females, according to the 2006 Guidelines on Preservation and Control Overweight and Obesity in Chinese Adults classification. ${ }^{15}$

\section{Peripheral artery blood pressure measurement}

Peripheral blood pressure was measured with a standard mercury sphygmomanometer, using the first and fifth Korotkoff sounds as the systolic and diastolic blood pressure, while the subjects were in a sedentary position, having rested for at least 5 minutes. The appropriate size cuff was determined based on arm circumference. Peripheral pulse pressure ( $\mathrm{pPP})$ is the difference between systolic and diastolic blood pressures.

\section{Arterial stiffness and central hemodynamics analyses}

Arterial stiffness was assessed by automated measurement of carotid-femoral pulse wave velocity (cfPWV) using a Complior Colson device (Créatech, Besançon, France), in the morning, in a quiet environment, at stable temperature. The technical characteristics of this device have been described previously. ${ }^{16}$ Participants were studied in the supine position, after resting for at least 5 minutes. PWV along the artery was measured using two strain gauge transducers (TY-306 pressure-sensitive transducer, Fukuda Denshi Co, Tokyo, Japan), fixed transcutaneously over the course of the arteries, separated by a known distance; the right-side carotid, femoral, and radial arteries were used. After waveforms of sufficient quality were recorded, the digitization process was initiated by the operator, and automatic calculation of the time delay between two upstrokes was begun. Measurements were repeated over ten cardiac cycles, and the mean value was used in the final analysis. PWV was calculated from the measurement of the pulse transit time and distance traveled by the pulse between the two recording sites (measured on the surface of the body, in meters), according to the following formula: PWV $(\mathrm{m} / \mathrm{s})=$ distance $(\mathrm{m}) /$ transit time $(\mathrm{s})$. The right radial artery pressure wave waveform was recorded using an instrument designed for such measurements (SphygmoCor, AtCor Medical Pty, West Ryde, NSW, Australia). The radial artery was gently compressed with the tip of a tonometer 
at the site of maximal pulsation. The tonometer contains a micromanometer, which provides a very accurate recording of the pressure within the artery. The system software was used to calculate the average radial artery waveform; the corresponding central artery pressure waveform and cPP were generated using a transfer function of the instrument.

\section{Laboratory analyses}

Blood samples were collected between 8 am and 10 am, after subjects had fasted for at least 12 hours. Blood samples were stored at $4^{\circ} \mathrm{C}$, and delivered to the central laboratory in the Department of Biochemistry, Chinese People's Liberation Army General Hospital on the same day. Serum fasting blood glucose (FBG), high density lipoprotein cholesterol (HDL-C), and low density lipoprotein cholesterol (LDLC) levels were determined using enzymatic assays (Roche, Basel, Switzerland), on a fully automatic biochemical autoanalyzer (Cobas ${ }^{\circledR} 6000$, Roche). The technicians who performed the different tests were blinded to the clinical data of the subjects.

\section{Statistical analyses}

Categorical variables were expressed as numbers and percentages. Continuous variables were described using means with standard deviations, for data with normal distribution, and medians with interquartile ranges, for nonnormally distributed data. The participants were stratified by the presence (or not) of overall and abdominal obesity. Simple comparison was made between groups using the Student's $t$-test for continuous variables (normal distribution), the Mann-Whitney $U$ test for continuous variables (non-normal distribution), and the $\chi^{2}$ test for categorical variables. Logistic regression analysis was used to compare variables between groups after adjustment for age and sex. We logarithmically transformed cfPWV and cPP to meet the multinormality assumption, then performed multiple linear regression analysis, using cfPWV and cPP, as dependent variables, and $\mathrm{BMI}$ and $\mathrm{WC}$, as independent variables, following two models. In the first model, age and sex were included as adjustment variables; and in the second model, age, sex, pPP, FBG, HDL-C, and LDL-C were included. All analyses were conducted using SPSS version 17 statistical software (IBM Corp, Armonk, NY, USA). A $P$-value $<0.05$ was considered statistically significant.

\section{Results}

The median age of all participants was 54 years, with an age range of 18-96 years; $51.8 \%$ were male. Median values of BMI and WC were $25.1 \mathrm{~kg} / \mathrm{m}^{2}$ and $86 \mathrm{~cm} ; 19.3 \%$ were overall obese and $63.1 \%$ were abdominally obese. Median values of cfPWV and cPP were $10.2 \mathrm{~m} / \mathrm{s}$ and $37 \mathrm{mmHg}$, respectively. Table 1 shows the demographic and clinical characteristics of all individuals, grouped by the presence (or not) of overall and abdominal obesity. Compared with non-obese individuals, both overall and abdominally obese individuals were older, with significantly higher cfPWV, cPP, pPP, FBG, and LDL-C levels, and lower HDL-C level $(P<0.001$, for all). The percentages of males were similar between overall obese and non-obese groups, and between abdominally obese and non-obese groups ( $P>0.05$, for all). Compared with non-obese individuals, abdominally obese individuals had significantly higher BMI, and overall obese individuals had significantly higher WC $(P<0.001$, for both). Compared with non-obese individuals, both overall

Table I Demographic and clinical characteristics of all adults, by presence of overall and abdominal obesity

\begin{tabular}{|c|c|c|c|c|c|c|c|c|}
\hline Characteristics & $\begin{array}{l}\text { Overall } \\
\text { non-obesity } \\
(n=2,1 \mid 7)\end{array}$ & $\begin{array}{l}\text { Overall } \\
\text { obesity } \\
(n=507)\end{array}$ & $P$-value ${ }^{a}$ & $P$-value ${ }^{b}$ & $\begin{array}{l}\text { Abdominal } \\
\text { non-obesity } \\
(n=969)\end{array}$ & $\begin{array}{l}\text { Abdominal } \\
\text { obesity } \\
(n=1,655)\end{array}$ & $P$-value ${ }^{a}$ & $P$-value \\
\hline Age (years) ${ }^{c}$ & $53(38-66)$ & $56(47-67)$ & $<0.001$ & & $46(33-60)$ & $58(46-68)$ & $<0.001$ & \\
\hline Males (\%) & I,II $3(52.6)$ & $245(48.3)$ & 0.085 & & $488(50.4)$ & $870(52.6)$ & 0.275 & \\
\hline BMI $\left(\mathrm{kg} / \mathrm{m}^{2}\right)^{\mathrm{c}}$ & $\begin{array}{l}24.34 \\
(22.38-25.95)\end{array}$ & $\begin{array}{l}29.69 \\
(28.67-31.26)\end{array}$ & $<0.001$ & & $\begin{array}{l}22.58 \\
(20.83-24.15)\end{array}$ & $\begin{array}{l}26.57 \\
(24.97-28.4 I)\end{array}$ & $<0.001$ & $<0.001$ \\
\hline$W C(\mathrm{~cm})^{c}$ & $83(77-89)$ & $96(91-101)$ & $<0.001$ & $<0.001$ & $76(7 I-79)$ & 90 (86-95) & $<0.001$ & \\
\hline PPP $(\mathrm{mmHg})^{c}$ & $47(40-58)$ & $50(42-62)$ & $<0.001$ & $<0.001$ & $45(38-54)$ & $50(40-60)$ & $<0.001$ & 0.004 \\
\hline $\mathrm{FBG}(\mathrm{mmol} / \mathrm{L})^{\mathrm{c}}$ & $4.91(4.54-5.35)$ & $5.09(4.64-5.70)$ & $<0.001$ & 0.016 & $4.81(4.49-5.16)$ & $5.03(4.63-5.60)$ & $<0.001$ & $<0.001$ \\
\hline HDL-C $(\mathrm{mmol} / \mathrm{L})^{c}$ & $1.38(1.17-1.64)$ & $1.22(1.05-\mid .4 I)$ & $<0.001$ & $<0.001$ & $1.52(1.29-1.79)$ & $1.26(1.08-1.47)$ & $<0.001$ & $<0.001$ \\
\hline LDL-C $(\mathrm{mmol} / \mathrm{L})^{c}$ & $2.70(2.23-3.21)$ & $2.95(2.42-3.34)$ & $<0.001$ & $<0.001$ & $2.50(2.03-3.02)$ & $2.89(2.40-3.34)$ & $<0.001$ & $<0.001$ \\
\hline $\operatorname{cfPWV}(\mathrm{m} / \mathrm{s})^{c}$ & I0.I (8.8-12.0) & $10.5(9.4-12.1)$ & $<0.001$ & 0.518 & $9.5(8.4-11.0)$ & $10.6(9.2-12.5)$ & $<0.001$ & 0.008 \\
\hline $\mathrm{cPP}(\mathrm{mmHg})^{\mathrm{c}}$ & $37(30-47)$ & $40(33-5 I)$ & $<0.001$ & $<0.001$ & $35(29-42)$ & $39(33-50)$ & $<0.001$ & 0.193 \\
\hline
\end{tabular}

Notes: a ${ }^{P}$-value not adjusted; ${ }^{b} P$-value adjusted for age and sex; ${ }^{c}$ median (interquartile range).

Abbreviations: BMI, body mass index; WC, waist circumference; pPP, peripheral pulse pressure; FBG, fasting blood glucose; HDL-C, high density lipoprotein cholesterol; LDL-C, low density lipoprotein cholesterol; cfPWV, carotid-femoral pulse wave velocity; cPP, central pulse pressure. 
obese and abdominally obese individuals had independently higher pPP, FBG, and LDL-C levels, and lower HDL-C level $(P<0.05$, for all), after adjusting for age and sex. More importantly, compared with non-obese individuals, overall obese individuals had independently higher cPP $(P<0.05)$, but not cfPWV $(P>0.05)$, after adjusting for age and sex, while abdominally obese individuals had independently higher cfPWV $(P<0.05)$, but not cPP $(P>0.05)$.

In multivariate association analysis (Table 2), in the first and second models, WC ( $P<0.05$, for both), rather than BMI ( $P>0.05$, for both) was independently associated with cfPWV. Although both BMI and WC were both independently associated with cPP in the first model ( $P<0.001$, for both), only BMI $(P<0.001)$, and not WC $(P>0.05)$, was independently associated with cPP in the second model. In addition, age, sex, pPP, FBG, and HDL-C levels ( $P<0.05$, for all), but not LDL-C level $(P>0.05)$, were independently associated with cfPWV. Meanwhile, age, sex, pPP ( $P<0.001$, for all), but not FBG, HDL-C, and LDL-C levels ( $P>0.05$, for all), had independent association with cPP.

\section{Discussion}

In Chinese community-dwelling adults, the current study demonstrated that $\mathrm{WC}$, rather than BMI, was correlated with central arterial stiffness, as assessed by cfPWV, independent of age, sex, blood pressure, glucose, and lipids, while BMI, rather than $\mathrm{WC}$, was independently associated with central hemodynamics, as measured by cPP. Moreover, age, sex, and blood pressure were independently associated with central arterial stiffness and hemodynamics, but blood glucose and lipids were independently associated with central arterial stiffness, rather than central hemodynamics.

Elevated central arterial stiffness and abnormal central hemodynamics reflect impairment of arterial structure and function and increased risk of cardiovascular disease, which leads to elevated mortality; ${ }^{5,6}$ hence, the measurements of central arterial stiffness and hemodynamics is beneficial to identify the cardiovascular damage. Although other methods exist to estimate central arterial stiffness and hemodynamics, PWV is widely used as the classical marker of central arterial stiffness,

Table 2 Multivariate association of pulse wave velocity and central pulse pressure with anthropometric measurements

\begin{tabular}{|c|c|c|c|c|c|c|}
\hline \multirow[t]{2}{*}{ Characteristics } & \multicolumn{3}{|l|}{ cfPWV } & \multicolumn{3}{|l|}{ cPP } \\
\hline & Standardized $\beta$ & $\mathbf{t}$ & $P$-value & Standardized $\beta$ & $\mathbf{t}$ & $P$-value \\
\hline \multicolumn{7}{|l|}{ BMI $\left(\mathrm{kg} / \mathrm{m}^{2}\right)$} \\
\hline \multicolumn{7}{|l|}{ First model ${ }^{\mathrm{a}}$} \\
\hline BMI $\left(\mathrm{kg} / \mathrm{m}^{2}\right)$ & 0.021 & 1.304 & 0.192 & 0.108 & 6.794 & $<0.001$ \\
\hline Age (years) & 0.588 & 35.361 & $<0.001$ & 0.564 & 34.571 & $<0.001$ \\
\hline Females/males & -0.123 & -7.44 I & $<0.001$ & 0.031 & 1.924 & 0.054 \\
\hline \multicolumn{7}{|l|}{ Second model ${ }^{b}$} \\
\hline BMI $\left(\mathrm{kg} / \mathrm{m}^{2}\right)$ & -0.020 & -1.203 & 0.229 & 0.063 & 4.311 & $<0.001$ \\
\hline Age (years) & 0.501 & 26.449 & $<0.001$ & 0.358 & 21.824 & $<0.001$ \\
\hline Females/males & -0.098 & -5.900 & $<0.00$ I & 0.054 & 3.729 & $<0.00 \mathrm{I}$ \\
\hline PPP $(\mathrm{mmHg})$ & 0.196 & 11.262 & $<0.001$ & 0.466 & 30.843 & $<0.001$ \\
\hline FBG (mmol/L) & 0.053 & 3.314 & 0.001 & -0.015 & -1.076 & 0.282 \\
\hline $\mathrm{HDL}-\mathrm{C}(\mathrm{mmol} / \mathrm{L})$ & -0.060 & -3.489 & $<0.001$ & -0.001 & -0.082 & 0.935 \\
\hline LDL-C (mmol/L) & -0.021 & -1.206 & 0.228 & 0.013 & 0.895 & 0.371 \\
\hline \multicolumn{7}{|l|}{$W C(\mathrm{~cm})$} \\
\hline \multicolumn{7}{|l|}{ First model ${ }^{\mathrm{a}}$} \\
\hline$W C(\mathrm{~cm})$ & 0.075 & 4.256 & $<0.001$ & 0.077 & 4.428 & $<0.001$ \\
\hline Age (years) & 0.563 & 31.865 & $<0.001$ & 0.551 & 31.511 & $<0.001$ \\
\hline Females/males & -0.101 & -5.823 & $<0.001$ & 0.048 & 2.798 & 0.005 \\
\hline \multicolumn{7}{|l|}{ Second model ${ }^{b}$} \\
\hline$W C(\mathrm{~cm})$ & 0.036 & 1.897 & 0.048 & 0.021 & 1.257 & 0.209 \\
\hline Age (years) & 0.492 & 25.212 & $<0.001$ & 0.354 & 20.893 & $<0.001$ \\
\hline Females/males & -0.090 & -5.245 & $<0.001$ & 0.060 & 3.981 & $<0.001$ \\
\hline pPP (mmHg) & 0.191 & 10.960 & $<0.001$ & 0.470 & 31.038 & $<0.001$ \\
\hline $\mathrm{FBG}(\mathrm{mmol} / \mathrm{L})$ & 0.050 & 3.148 & 0.002 & -0.014 & -1.006 & 0.315 \\
\hline HDL-c (mmol/L) & $-0.04 I$ & -2.335 & 0.020 & -0.016 & -1.078 & 0.281 \\
\hline LDL-c (mmol/L) & -0.027 & $-1.59 \mid$ & 0.112 & 0.018 & 1.208 & 0.227 \\
\hline
\end{tabular}

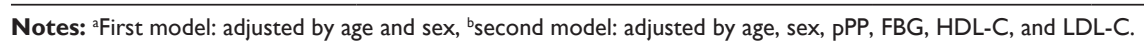

Abbreviations: cfPWV, carotid-femoral pulse wave velocity; cPP, central pulse pressure; BMI, body mass index; WC, waist circumference; pPP, peripheral pulse pressure; FBG, fasting blood glucose; HDL-C, high density lipoprotein cholesterol; LDL-C, low density lipoprotein cholesterol. 
while cPP is well regarded as a non-invasive indicator of central hemodynamics. ${ }^{?}$

It is well known that metabolic disorders, including adiposity, can have an adverse influence on arterial structure and function. A line of studies has researched the relationship between obesity and central arterial stiffness and hemodynamics. ${ }^{8-11}$ For example, similar relationship between aortic stiffness and body weight has been documented in both the Cardiovascular Health Study and Health ABC study. ${ }^{12,13}$ However, limited studies have been dedicated to comparing overall and abdominal obesity in relation to central arterial stiffness and hemodynamics. A study of 305 Spanish subjects, by Recio-Rodriguez et al, reminded us that WC correlated better than BMI with arterial stiffness, as measured by PWV. ${ }^{14}$ The study had a small sample size, and was directed mainly toward hypertensive and diabetic subjects. Scarce large sample studies have elucidated whether the relationship might differ in Chinese community-dwelling adults. Previous studies have demonstrated that many Asians have different features of body shape, and those with lower BMI levels have higher amounts of body fat. ${ }^{17}$ But studies analyzing the relationship of obesity with central arterial stiffness and hemodynamics have been based on data from United States or European countries, with little data available on populations in the People's Republic of China. Thus, there is a need for a large sample investigation in Chinese community-dwelling adults paying close attention to the comparison of overall and abdominal obesity in relation to central arterial stiffness and hemodynamics. The current study demonstrated that the measure of abdominal obesity (WC) rather than the overall obesity indicator (BMI) was associated with arterial stiffness, as evaluated by cfPWV, independent of age, sex, blood pressure, glucose, and lipids, while the overall obesity indicator (BMI), but not the abdominal obesity indicator (WC), was independently correlated with central hemodynamics, as evaluated by cPP.

There are a number of reasons why abdominal obesity might be more relevant to central arterial stiffening, as compared to overall obesity. First, insulin resistance has been shown to accompany abdominal obesity. ${ }^{18,19}$ Insulin resistance likely has vascular effects through associated hyperinsulinemia and increased glycemia. The effects of hyperinsulinemia on the vascular system might include promotion of sodium reabsorption, ${ }^{20}$ stimulation of the sympathetic nervous system, ${ }^{21}$ and promotion of vascular smooth muscle cell growth, ${ }^{22}$ all of which might contribute to increased central arterial stiffness. The second mechanism by which abdominal obesity might contribute to central arterial stiffening is through inflammation, which has been shown to be related to abdominal obesity. ${ }^{23}$ The presence of higher levels of circulating immune system cells possibly promotes their movement into the artery wall, leading to wall stiffening. Furthermore, sufficient attention must be paid to the shared genetic and epigenetic relationship between different types of obesity and central arterial stiffness and hemodynamics. The reason why overall obesity has a more significant relationship with central hemodynamics than abdominal obesity is not clear. Further experimental and clinical studies are needed.

Some limitations of the current study must be considered. First, due to its cross-sectional design, the study was inherently limited in its ability to determine causal relationship between different obesity indicators and central arterial stiffness and hemodynamics; further prospective, population-based studies are needed to investigate causal mechanisms. Second, since some of the study population might take different types of drugs, the current study failed to observe possible effects of drugs on central arterial stiffness and hemodynamics.

Based on the results obtained in our study, it can be concluded that in Chinese community-dwelling adults, the measure of abdominal obesity (WC), but not the overall obesity indicator (BMI), was related to central arterial stiffness, as represented by cfPWV, independent of age, sex, blood pressure, glucose, and lipids, while the overall obesity indicator (BMI), but not the abdominal obesity indicator (WC), was independently correlated with central hemodynamics, as measured by cPP. Furthermore, age, sex, and blood pressure were independently associated with central arterial stiffness and hemodynamics; and blood glucose and lipids were independently associated with central arterial stiffness, but not central hemodynamics.

\section{Acknowledgments}

This study was supported by grants from the National Key Basic Research Project (2012CB517503 and 2013CB530804), the Health Special Scientific Research Subject of Chinese People's Liberation Army (12BLZ34), and the Clinical Scientific Research Project of Chinese People's Liberation Army General Hospital (2012FC-TSYS1021).

\section{Disclosure}

The authors declare that they have no conflicts of interest in this work.

\section{References}

1. Loehr LR, Rosamond WD, Poole C, et al. Association of multiple anthropometrics of overweight and obesity with incident heart failure: the Atherosclerosis Risk in Communities study. Circ Heart Fail. 2009;2: $18-24$. 
2. Turkbey EB, McClelland RL, Kronmal RA, et al. The impact of obesity on the left ventricle: the Multi-Ethnic Study of Atherosclerosis (MESA). JACC Cardiovasc Imaging. 2010;3:266-274.

3. Cheriyath P, Duan Y, Qian Z, et al. Obesity, physical activity and the development of metabolic syndrome: the Atherosclerosis Risk in Communities study. Eur J Cardiovasc Prev Rehabil. 2010;17: 309-313.

4. Hu YH, Reilly KH, Liang YJ, et al. Increase in Body Mass Index, Waist Circumference and Waist-to-height Ratio is Associated with High Blood Pressure in Children and Adolescents in China. J Int Med Res. 2011;39:23-32.

5. Laurent S, Boutouyrie P, Asmar R, et al. Aortic stiffness is an independent predictor of all-cause and cardiovascular mortality in hypertensive patients. Hypertension. 2001;37:1236-1241.

6. O'Rourke MF, Franklin SS. Arterial stiffness: reflections on the arterial pulse. Eur Heart J. 2006;27:2497-2498.

7. Lehmann ED. Clinical value of aortic pulse-wave velocity measurement. Lancet. 1999;354:528-529.

8. Ko MJ, Kim MK, Shin J, et al. Relations of pulse wave velocity to waist circumference independent of hip circumference. Epidemiol Health. 2010;32:e2010004.

9. Rider OJ, Tayal U, Francis JM, et al. The effect of obesity and weight loss on aortic pulse wave velocity as assessed by magnetic resonance imaging. Obesity (Silver Spring). 18, 2010:2311-2316.

10. Wildman RP, Mackey RH, Bostom A, et al. Measures of obesity are associated with vascular stiffness in young and older adults. Hypertension. 2003;42:468-473.

11. Ferreira I, Snijder MB, Twisk JW, et al. Central fat mass versus peripheral fat and lean mass: opposite (adverse versus favorable) associations with arterial stiffness? The Amsterdam Growth and Health Longitudinal Study. J Clin Endocrinol Metab. 2004;89: 2632-2639.

12. Sutton-Tyrrell K, Newman A, Simonsick EM, et al. Aortic stiffness is associated with visceral adiposity in older adults enrolled in the study of health, aging, and body composition. Hypertension. 2001;38: 429-433.
13. Mackey RH, Sutton-Tyrrell K, Vaitkevicius PV, et al. Correlates of aortic stiffness in elderly individuals: a subgroup of the Cardiovascular Health Study. Am J Hypertens. 2002;15:16-23.

14. Recio-Rodriguez JI, Gomez-Marcos MA, Patino-Alonso MC, et al. Abdominal obesity vs general obesity for identifying arterial stiffness, subclinical atherosclerosis and wave reflection in healthy, diabetics and hypertensive. BMC Cardiovasc Disord. 2012;12:3.

15. Hu J, Wallace DC, Jones E, et al. Cardiometabolic health of Chinese older adults with diabetes living in Beijing, China. Public Health Nurs. 2009;26:500-511.

16. Asmar R, Benetos A, Topouchian J, et al. Assessment of arterial distensibility by automatic pulse wave velocity measurement. Validation and clinical application studies. Hypertension. 1995;26:485-490.

17. Kagawa M, Kerr D, Uchida H, et al. Differences in the relationship between BMI and percentage body fat between Japanese and Australian-Caucasian young men. Br J Nutr. 2006;95:1002-1007.

18. Wahrenberg H, Hertel K, Leijonhufvud BM, et al. Use of waist circumference to predict insulin resistance: retrospective study. $B M J$. 2005;330:1363-1364.

19. Lemieux I, Pascot A, Couillard C, et al. Hypertriglyceridemic waist: A marker of the atherogenic metabolic triad (hyperinsulinemia; hyperapolipoprotein B; small, dense LDL) in men? Circulation. 2000;102: 179-184.

20. ter Maaten JC, Bakker SJ, Serne EH, et al. Insulin's acute effects on glomerular filtration rate correlate with insulin sensitivity whereas insulin's acute effects on proximal tubular sodium reabsorption correlate with salt sensitivity in normal subjects. Nephrol Dial Transplant. 1999; 14:2357-2363.

21. Young JB. Effect of experimental hyperinsulinemia on sympathetic nervous system activity in the rat. Life Sci. 1988;43:193-200.

22. Begum N, Song Y, Rienzie J, et al. Vascular smooth muscle cell growth and insulin regulation of mitogen-activated protein kinase in hypertension. Am J Physiol. 1998;275:C42-C49.

23. Rana JS, Arsenault BJ, Després JP, et al. Inflammatory biomarkers, physical activity, waist circumference, and risk of future coronary heart disease in healthy men and women. Eur Heart J. 2011;32:336-344.
Clinical Interventions in Aging

\section{Publish your work in this journal}

Clinical Interventions in Aging is an international, peer-reviewed journal focusing on evidence-based reports on the value or lack thereof of treatments intended to prevent or delay the onset of maladaptive correlates of aging in human beings. This journal is indexed on PubMed Central, MedLine, the American Chemical Society's 'Chemical Abstracts

\section{Dovepress}

Service' (CAS), Scopus and the Elsevier Bibliographic databases. The manuscript management system is completely online and includes a very quick and fair peer-review system, which is all easy to use. Visit $\mathrm{http}: / /$ www.dovepress.com/testimonials.php to read real quotes from published authors. 